\title{
p53 deficiency augments nucleolar instability after ionizing irradiation
}

\author{
SANGEETA KAKOTI $^{1,2}$, MOTOHIRO YAMAUCHI $^{3}$, WENCHAO GU $^{1}$, REONA KATO $^{4}$, TAKAAKI YASUHARA ${ }^{4}$, \\ YOSHIHIKO HAGIWARA ${ }^{2}$, SIDDHARTHA LASKAR ${ }^{5}$, TAKAHIRO OIKE ${ }^{2}$, HIRO SATO ${ }^{2}$, \\ KATHRYN D. HELD ${ }^{6,7}$, TAKASHI NAKANO ${ }^{2}$ and ATSUSHI SHIBATA ${ }^{1}$ \\ ${ }^{1}$ Gunma University Initiative for Advanced Research (GIAR); ${ }^{2}$ Department of Radiation Oncology, Gunma University \\ Graduate School of Medicine, Maebashi, Gunma 371-8511; ${ }^{3}$ Department of Radiation Biology and Protection, \\ Atomic Bomb Disease Institute, Nagasaki University, Nagasaki 852-8523; ${ }^{4}$ Laboratory of Molecular Radiology, \\ Center for Disease Biology and Integrative Medicine, Graduate School of Medicine, The University of Tokyo, \\ Tokyo 113-8655, Japan; ${ }^{5}$ Department of Radiation Oncology, Tata Memorial Hospital, Mumbai 400012, India; \\ ${ }^{6}$ Department of Radiation Oncology, Massachusetts General Hospital/Harvard Medical School, \\ Boston, MA 02114, USA; 7 International Open Laboratory, Gunma University Initiative \\ for Advanced Research (GIAR), Maebashi, Gunma 371-8511, Japan
}

Received March 22, 2019; Accepted August 20, 2019

DOI: $10.3892 /$ or.2019.7341

\begin{abstract}
Ribosomes are important cellular components that maintain cellular homeostasis through overall protein synthesis. The nucleolus is a prominent subnuclear structure that contains ribosomal DNA (rDNA) encoding ribosomal RNA (rRNA), an essential component of ribosomes. Despite the significant role of the rDNA-rRNA-ribosome axis in cellular homeostasis, the stability of rDNA in the context of the DNA damage response has not been fully investigated. In the present study, the number and morphological changes of nucleolin, a marker of the nucleolus, were examined following ionizing radiation (IR) in order to investigate the impact of DNA damage on nucleolar stability. An increase in the number of nucleoli per cell was found in HCT116 and U2OS cells following IR. Interestingly, the IR-dependent increase in nucleolar fragmentation was enhanced by p53 deficiency. In addition, the morphological analysis revealed several distinct types of nucleolar fragmentation following IR. The pattern of nucleolar morphology differed between HCT116 and U2OS cells, and the p53 deficiency altered the pattern of nucleolar morphology. Finally, a significant decrease in rRNA synthesis was observed in HCT116 $p 53^{-/-}$cells following IR, suggesting that severe nucleolar fragmentation downregulates rRNA transcription. The findings of the present study suggest
\end{abstract}

Correspondence to: $\mathrm{Dr}$ Atsushi Shibata, Gunma University Initiative for Advanced Research (GIAR), 3-39-22, Showa-machi, Maebashi, Gunma 371-8511, Japan

E-mail: shibata.at@gunma-u.ac.jp

Key words: rDNA gene expression, chromosome rearrangement, cancer development that p53 plays a key role in protecting the transcriptional activity of rDNA in response to DNA damage.

\section{Introduction}

The nucleolus is an intra-nuclear organelle that contains ribosomal DNA (rDNA) that encodes ribosomal RNA (rRNA), which is an essential component of ribosomes. The rRNA and a variety of ribosomal proteins form ribosomes, which serve as the site of biological protein synthesis. Previous studies have demonstrated that ribosome biogenesis is associated with multiple cellular activities. For example, limiting ribosome biogenesis extends the lifespan, whereas increased ribosome biogenesis has been reported as a hallmark of premature aging (1). In the context of cancer, alterations in ribosome biogenesis have been suggested to be involved in tumorigenesis by downregulating tumor suppressors $(2,3)$. In addition, rDNA rearrangements have been observed in over half of adult solid tumors (4). This observation suggests that rDNA restructuring is one of the most common chromosomal alterations in adult solid tumors and may be involved in tumorigenesis or malignancy. Conversely, for cancer treatment, an inhibitor targeting RNA polymerase I, which is required for rDNA transcription, induces apoptosis in cancer cells by activating the ATM/ATR pathway (5).

Ionizing radiation (IR) generates multiple types of DNA damage, such as DNA double-strand breaks (DSBs), single-strand breaks (SSBs), and base damage (6). Among the various types of DNA damage, DSBs are considered to be a critical factor causing severe genome instability, such as deletion, duplication, inversion and translocation (7). Linear energy transfer (LET) is a parameter used to describe the pattern of energy deposition. X-rays or $\gamma$-rays are known as low LET IR, whereas heavy charged particle IR is high LET radiation. High LET particle irradiation causes complex DNA lesions, defined 
as DNA damage containing both DSBs and SSBs, as well as base damage, within 1-2 helical turns $(<3-4 \mathrm{~nm})(8)$.

Another feature of high LET particle irradiation-dependent DNA damage was recently identified, observed as clustered $\gamma \mathrm{H} 2 \mathrm{AX}$ foci along the particle track (9-11). A combination of complex DNA lesions within clustered DSBs is not efficiently repaired and is associated with a high cell-killing effect $(9,12)$. IR is a critical risk factor for human health, particularly for astronauts during periods of space travel or when radiation accidents occur, whereas it is significantly beneficial for cancer treatment (13).

In human cells, $150-200$ copies of rDNA encoding rRNA is located on chromosomes $13,14,15,21$ and 22 , and each chromosome contains repetitive rDNA sequences (14). These repetitive sequences are genetically unstable and highly sensitive when cells are exposed to exogenous DNA stress (15). Classical studies have demonstrated that morphological changes in the nucleolus have been observed following IR under a phase-contrast microscope (16). However, the stability of the nucleolus and the transcriptional activity of rDNA following IR in the context of DSBs and its signaling have not been thoroughly investigated. Moreover, although irradiation-induced dose-dependent increase in polysomal aggregates has been reported (17), the irradiation-dependent morphological changes of individual ribosomes have not been investigated.

The aim of the present study was to examine the alterations in the amount of nucleolin, a marker of the nucleolus, and its morphological changes following IR, in order to investigate nucleolar stability in response to DSBs. We also investigated the role of p53 in preventing nucleolar instability following IR and protecting the transcriptional activity of rDNA in irradiated cells.

\section{Materials and methods}

Cell culture and irradiation. HCT116 (human colorectal carcinoma, wild-type (WT) $p 53^{+/+}$and isogenic $p 53^{-/-}$null derivative, provided by Dr Vogelstein of Johns Hopkins University) and U2OS (human osteosarcoma) cells were cultured in Eagle's minimum essential medium (MEM) with $10 \%$ fetal calf serum (FCS) (Sigma-Aldrich; Merck KGaA). 1BR (normal human dermal fibroblast strain) hTERT cells were cultured in the alpha modification of MEM (Wako Pure Chemical Industries, Ltd.) with $10 \%$ FCS. X-ray irradiation was performed using a Faxitron RX-650 at $100 \mathrm{kVp}$ and $20 \mathrm{~mA}$ with a dose rate of $1.14 \mathrm{~Gy} / \mathrm{min}$ (Faxitron Bioptics). Carbon ion irradiation was performed at Gunma University Heavy Ion Medical Center using a spread-out Bragg-peak (SOBP) beam (290 MeV/nucleon; mean LET at the center of a 6-cm SOBP, $50 \mathrm{keV} / \mu \mathrm{m}$ ).

siRNA knockdown and p53 overexpression. In U2OS cells, p53 siRNA transfection was performed using HiPerFect (Qiagen $\mathrm{GmbH}$ ), as previously described (18). The efficiency of the knockdown was confirmed by immunoblotting for the detection of p53. The sequence of the p53 siRNA oligonucleotide used was 5'-GUGCAGCUGUGGGUUGAUUdTdT-3'. To examine the effect of p53 overexpression on nucleolar stability after IR, Flag-p53 WT was transfected in HCT116 cells using the Neon ${ }^{\circledR}$ Transfection System (Thermo Fisher Scientific,
Inc.). Transfection efficiency was confirmed by immunoblotting using p53 antibodies (1:500 anti-p53 antibody, sc-6243, Santa Cruz Biotechnology, Inc.) and flag (1:1,000 anti-flag antibody, F3165, Sigma-Aldrich; Merck KGaA). Cells were incubated in fresh MEM for $24 \mathrm{~h}$ and then irradiated and processed for immunofluorescence analysis.

Immunofluorescence staining. Cells were seeded on Fisherbrand microscope cover glasses (12-545-80 12CIR-1, Thermo Fisher Scientific, Inc.) $48 \mathrm{~h}$ prior to irradiation. At the indicated time points after irradiation, cells were fixed with $3 \%$ paraformaldehyde-2\% sucrose for $10 \mathrm{~min}$. After washing with phosphate-buffered saline (PBS), permeabilization was carried out by treatment with $0.2 \%$ TritonX-100-PBS (Sigma-Aldrich; Merck $\mathrm{KGaA}$ ) for $2 \mathrm{~min}$ and $30 \mathrm{sec}$. Cells were then washed twice with PBS and incubated with the primary antibody in $2 \%$ bovine serum albumin (BSA)-PBS (1:1,000 anti-nucleolin antibody, ab22758, Abcam) at $37^{\circ} \mathrm{C}$ for $30 \mathrm{~min}$. The cells were washed twice with PBS before being incubated with the appropriate secondary antibody conjugated to Alexa-Fluor-555 (1:500 in $2 \% \mathrm{BSA}-\mathrm{PBS}$ ) at $37^{\circ} \mathrm{C}$ for $30 \mathrm{~min}$. The cells were again washed twice with PBS and mounted onto slides using ProLong Gold antifade mountant (Thermo Fisher Scientific, Inc.).

Morphological evaluation of nucleoli. Immunofluorescence slides were examined with a Nikon microscope (ECLIPSE Ni-U with DS-Qi2 camera and NIS-Elements D) using a 60x magnification lens. From a representative area of each sample, at least 100 consecutive cells were evaluated by direct visualization. First, each cell was examined simultaneously for nuclear staining and nucleolar morphology, and then categorized into one of the seven nucleolar types. The number of nucleoli per cell was noted for all cells, excluding type 7 cells (cells undergoing apoptosis, mitosis, or mitotic catastrophe). Nucleolin signal within micronuclei (types 3 and 6) were also counted individually, and the total number was a combination of the signal within the main nuclei and micronuclei.

Quantification of $47 S$ rRNA expression levels using reverse transcription-quantitative polymerase chain reaction $(R T-q P C R)$ analysis. Cells were seeded and incubated for $24 \mathrm{~h}$ prior to irradiation. At $72 \mathrm{~h}$ of irradiation, total RNA was extracted from the cells using NucleoSpin RNA (Macherey-Nagel GmbH). PrimeScript RT Reagent Kit (Perfect Real Time; Takara) was used to reverse-transcribe cDNA from total RNA, according to the manufacturer's instructions, and qPCR was performed using StepOnePlus (Thermo Fisher Scientific, Inc.). Reactions (20 $\mu \mathrm{l}$ each) were prepared in duplicate in a MicroAmp Fast Optical 96-Well Reaction Plate (Applied Biosystems; Thermo Fisher Scientific, Inc.). Each reaction contained $0.5 \mu \mathrm{M}$ of each primer, $0.2 \mu \mathrm{M}$ probe, $10 \mu \mathrm{l}$ of TaqMan Universal PCR Master Mix (Applied Biosystems; Thermo Fisher Scientific, Inc.), and cDNA as a template. The expression levels were normalized to $G A P D H$ and were calculated using the $2^{-\triangle \Delta \mathrm{Cq}}$ method (19). The qPCR settings were as follows: Initial denaturation at $95^{\circ} \mathrm{C}$ for $10 \mathrm{~min}$, followed by 45 cycles of denaturation at $95^{\circ} \mathrm{C}$ for $15 \mathrm{sec}$, and annealing and extension at $60^{\circ} \mathrm{C}$ for $1 \mathrm{~min}$. The primers and probes used for the qPCR are listed below: 

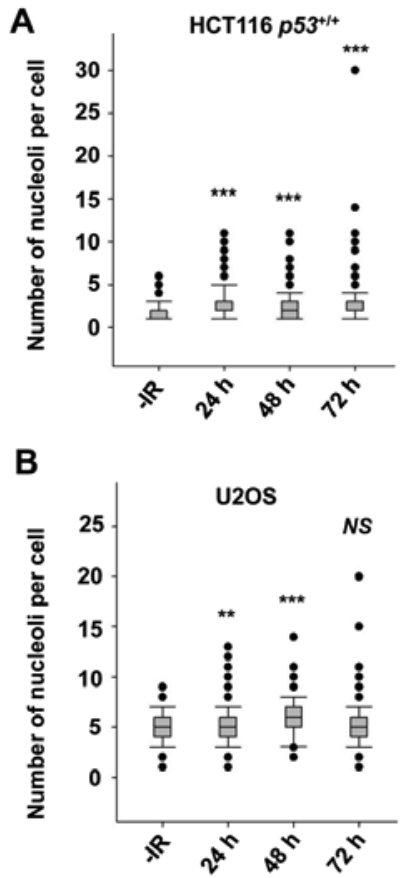

C

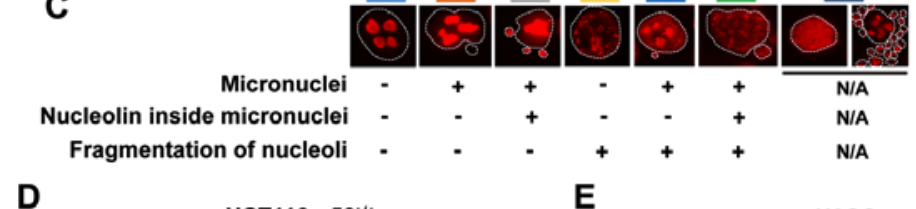

D

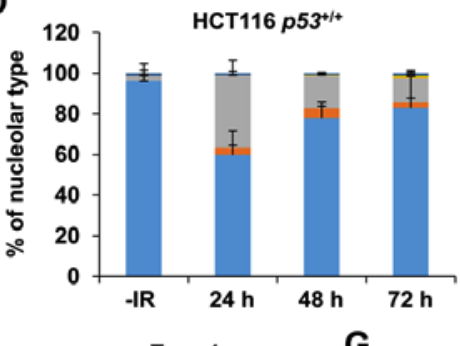

E
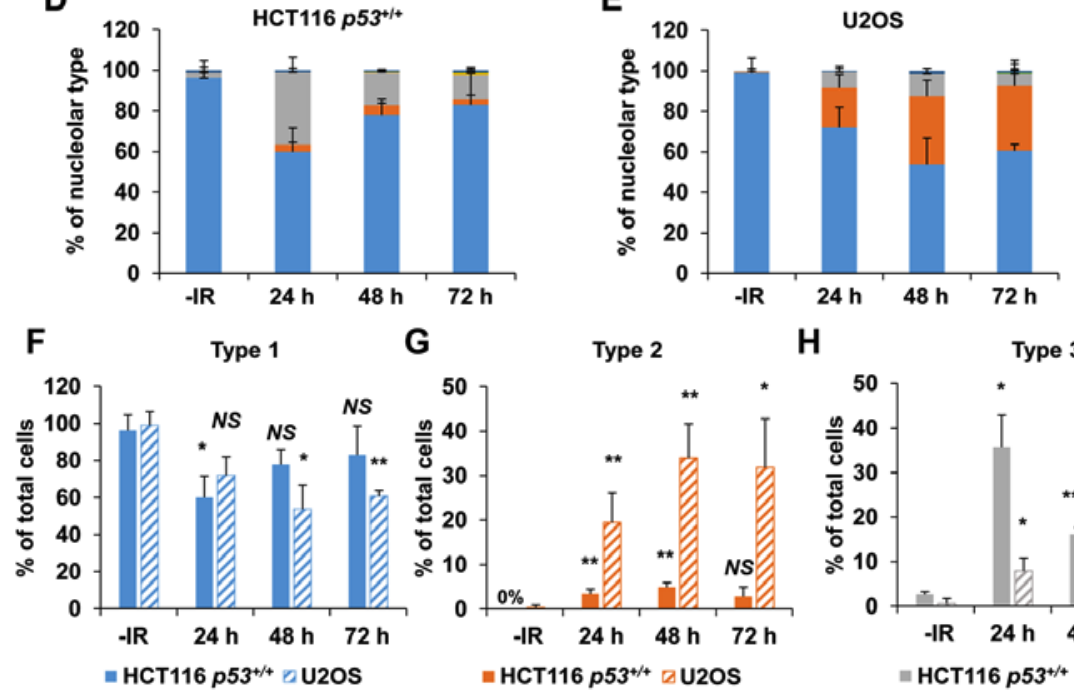

$\mathrm{H}$

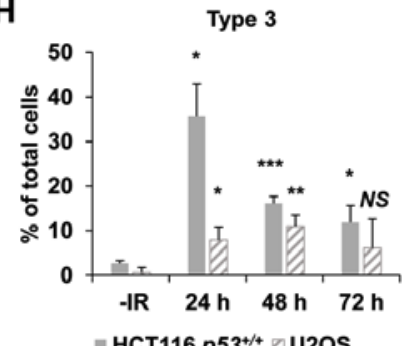

Figure 1. X-rays cause nucleolar fragmentation in human cancer cell lines. (A and B) Distribution of the number of nucleoli per cell at 24,48 and $72 \mathrm{~h}$ after irradiation with 2 Gy of X-rays in (A) HCT116 $p 53^{+/+}$and (B) U2OS cells. Box plot: Upper horizontal line of the box, 75th percentile; lower horizontal line of the box, 25th percentile; horizontal line within the box, median; upper horizontal bar outside the box, highest observation; lower horizontal bar outside the box, smallest observation. Circles represent outliers. (C) Cells were categorized into seven types based on the morphological characteristics of the nuclei (presence of micronuclei, as seen by DAPI staining corresponding to the white dashed line) and nucleoli (presence of fragmentation and localization inside micronuclei, if the latter is present). Type 7 includes apoptotic, mitotic, and mitotic catastrophe cells, which were subsequently excluded from the nucleolar quantification. (D and E) Percentage of nucleolar types in the total set of evaluated cells as shown in (D) HCT116 p53 $3^{+/+}$and (E) U2OS cells. Cells were analyzed at 24,48 and $72 \mathrm{~h}$ after irradiation with 2 Gy of X-rays. (F-H) Percentage of predominant individual nucleolar types. Types (F) 1 , (G) 2 and (H) 3 in the total set of evaluated HCT116 $\mathrm{p5}^{+/+}$and U2OS cells at 24, 48 and $72 \mathrm{~h}$ after irradiation with 2 Gy of X-rays. The error bars represent the standard deviation of three independent experiments. The statistical significance in A, B and F-H was examined by comparison with non-irradiated cells of the corresponding cell line, using Bonferroni's correction. ${ }^{*} \mathrm{P}<0.0167,{ }^{* *} \mathrm{P}<0.0033,{ }^{* * *} \mathrm{P}<0.0003$. NS, not significant; IR, ionizing radiation.

47S rRNA forward: 5'-GAACGGTGGTGTGTCGTTC-3' 47S rRNA reverse: 5'-GCGTCTCGTCTCGTCTCACT-3' 47S rRNA probe: 5'-CGGTGTGGGGTTCGAGGCGGTTT-3' GAPDH forward: 5'-CTCCTCTGACTTCAACAGCGA-3' GAPDH reverse: 5'-CCAAATTCGTTGTCATACCAGGA-3' GAPDH probe: 5'-ATGCCAGCCCCAGCGTCAAAGGT-3'

Statistical analysis. Box plots were constructed using SigmaPlot 12.3 (Systat Software, Inc.) from the combined data of three independent experiments. The statistical significance was analyzed using the Student's two-tailed t-test or Mann-Whitney U test. For datasets where multiple comparisons were performed using single group, to avoid the high rate of familywise error, Bonferroni's correction was applied. The level of significance was determined using the $\alpha$-value obtained by Bonferroni's correction and is specified in each individual figure legend.

\section{Results}

$X$-rays cause nucleolar fragmentation in human cancer cell lines. To investigate the nucleolar stability in response to IR in cancer cells, the number of nucleoli per cell and its morphological changes were examined following X-ray irradiation (n.b., nucleolin is localized within the nucleolus; therefore, it is used as a marker of nucleolus). In HCT116 $p 53^{+/+}$and U2OS cell lines, there was an increase in the number of nucleoli per cell over time after 2 Gy of X-ray irradiation (Fig. 1A and B). Next, the morphological patterns were categorized to investigate the morphological changes after IR (Fig. 1C). Interestingly, a marked increase in type $3 \mathrm{HCT} 116 \mathrm{p} 53^{+/+}$cells was observed following IR (Fig. 1D). Type 3 represents a cell containing micronuclei with a nucleolin signal. The peak of type 3 among HCT116 $p 53^{+/+}$cells was observed at $24 \mathrm{~h}$ following IR. By contrast, U2OS cells exhibited an increase in types 2 and 3 following X-ray irradiation (Fig. 1E). The majority of the nucleolar aberrations in U2OS cells were type 2 (cell containing micronuclei without nucleolin signal). These data suggest that nucleolar instability is caused in cancer cells following IR, and the type of morphological changes is cell type-dependent (Fig. 1F-H, supplementary information Fig. S1A). Furthermore, the levels of nucleolar instability were examined after different doses of X-ray irradiation. Importantly, the incidence of nucleolar fragmentation and type 3 morphology increased with the dose of X-ray irradiation until it peaked at 2-4 Gy, followed by a modest decline at $>8$ Gy at all the evaluated time points (Fig. 2A and B).

Next, the number of nucleoli in normal human fibroblasts was examined following IR to investigate the nucleolar stability in normal cells. In contrast to the observation of the increase in 

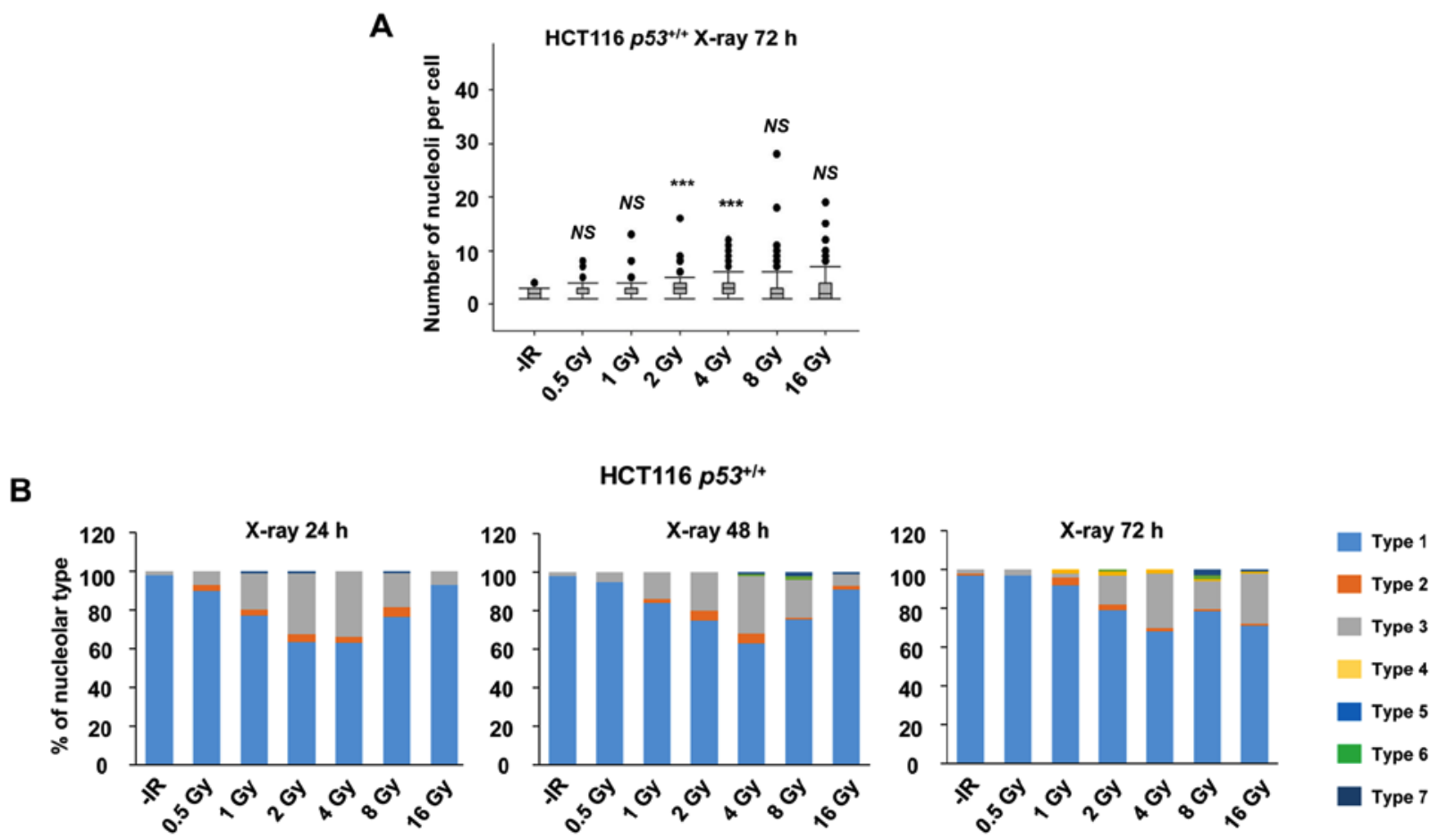

Figure 2. X-rays cause dose-dependent nucleolar fragmentation. (A) Distribution of the number of nucleoli per cell at $72 \mathrm{~h}$ after irradiation with $0.5,1,2,4$, 8, or 16 Gy of X-rays in HCT116 $p 53^{+/+}$cells. (B) Percentage of nucleolar types in the total set of evaluated cells after $0.5,1,2,4,8$ and 16 Gy of X-rays at 24 , 48 and $72 \mathrm{~h}$ after irradiation in HCT116 $p 53^{+++}$cells. Similar results were obtained in two independent experiments. Statistical significance was examined by comparison of samples irradiated with different doses individually with non-irradiated cells, using Bonferroni's correction. ${ }^{* * *} \mathrm{P}<0.00017$. NS, not significant; IR, ionizing radiation.

the number of nucleoli in cancer cell lines, X-ray irradiation did not increase the number of nucleoli in normal human fibroblasts, even at markedly higher radiation doses (Fig. 3).

p53 deficiency augments nucleolar fragmentation following exposure to $X$-rays. Furthermore, to address whether the genetic status involving genome stability affects nucleolar fragmentation after IR, the number of nucleoli per cell and any morphological changes were examined in cancer cells with p53 deficiency. Then, we compared the nucleolin signal in HCT116 $p 53^{+/+}$and HCT116 $p 53^{-/-}$cells to investigate the impact of the $p 53$ gene status on nucleolar stability. Importantly, a statistically significant $(\mathrm{P}=0.003)$ increase in nucleolar number was observed in HCT116 $p 53^{-/-}$cells at $72 \mathrm{~h}$ following exposure to X-rays (Fig. 4A). Furthermore, a dose-dependent increase in the nucleolar number of HCT116 $p 53^{--}$cells up to 8 Gy was found at $72 \mathrm{~h}$ (Fig. 4B). To investigate the effect of p53 on U2OS cells, siRNA knockdown targeting p53 was performed in U2OS cells. Similar to the results in HCT116 cells, there was a significant increase in the number of nucleoli in p53-depleted U2OS cells (Fig. 4C), with this increase being evident at later time points of $48-72 \mathrm{~h}$ after $\mathrm{X}$-ray irradiation.

Next, morphological changes following the categorization shown in Fig. 1C in p53-defective cells were analyzed following IR. A morphological categorization analysis revealed the appearance of type 4 morphology (multiple nucleolar fragmentations without micronuclei) in HCT116 $p 53^{-/-}$cells at $72 \mathrm{~h}$ (Fig. 4D, supplementary information Figs. S1B and S2A-E). In addition, dose-dependent analysis confirmed more

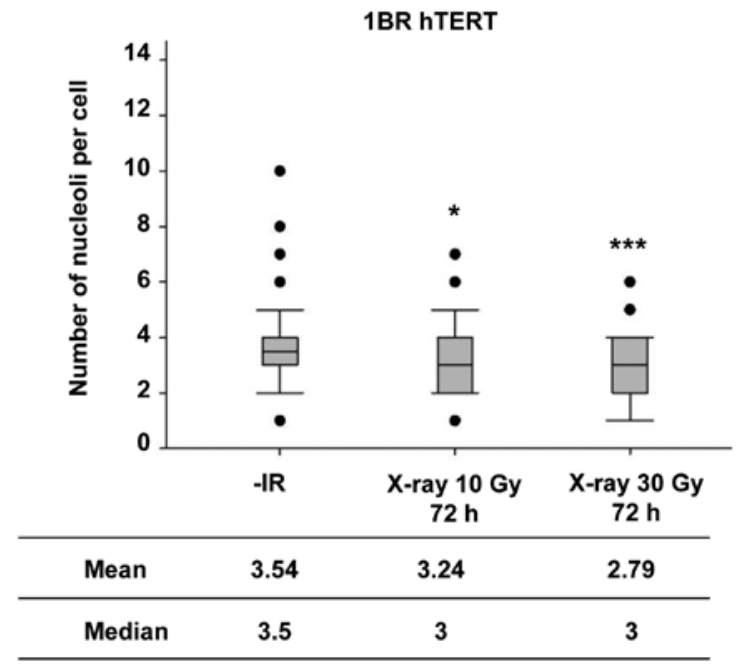

Figure 3. IR does not increase the number of nucleoli in normal human fibroblasts. Distribution of the number of nucleoli per cell in 1BR hTERT cells at $72 \mathrm{~h}$ following irradiation with 10 or $30 \mathrm{~Gy}$ of X-rays. The statistical significance was examined by comparison with non-irradiated cells, using Bonferroni's correction. ${ }^{*} \mathrm{P}<0.025,{ }^{* * *} \mathrm{P}<0.0005$. IR, ionizing radiation.

prominent type 4, type 6 and type 7 characteristics in HCT116 $p 53^{--}$cells (Fig. 4E). Alternatively, the morphological analysis in p53-depleted U2OS cells revealed a change in the nucleolar structure from type 2 to types 3 and 7 (Fig. 4F, supplementary information Figs. S1B and S2F-J). These results suggest that p53 is an important gene for suppressing nucleolar fragmentation following X-rays. 

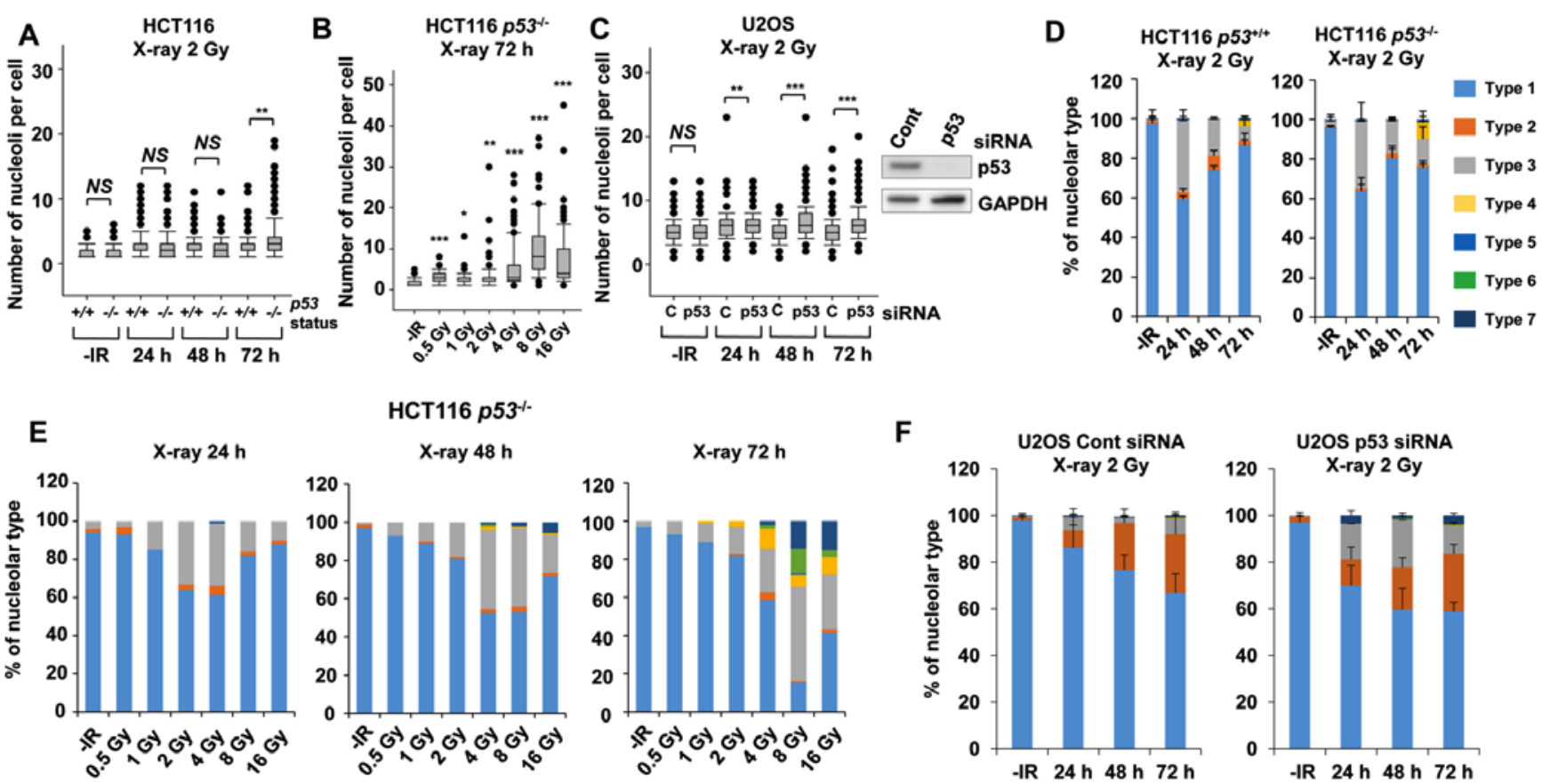

Figure 4. p53 deficiency augments nucleolar fragmentation following X-rays. (A) The effect of p53 deficiency was compared between $p 53^{+/+}$and $p 53^{-/-}$variants of HCT116 cells. Cells were irradiated with $2 \mathrm{~Gy}$ of X-rays and the distribution of the number of nucleoli per cell at 24,48 and $72 \mathrm{~h}$ after irradiation is shown. (B) Distribution of the number of nucleoli per cell at $72 \mathrm{~h}$ after irradiation with $0.5,1,2,4,8$, or 16 Gy of X-rays in HCT116 p53 ${ }^{-/}$cells. Statistical significance was examined by comparison with non-irradiated cells, using Bonferroni's correction. ${ }^{~} \mathrm{P}<0.0083,{ }^{* * *} \mathrm{P}<0.0017,{ }^{* * * *} \mathrm{P}<0.00017$. n.s., not significant (C) Distribution of the number of nucleoli per cell at 24, 48 and $72 \mathrm{~h}$ after irradiation in p53-depleted U2OS cells. The knockdown efficiency is shown in the right panel. C: siControl, p53: siRNA of p53. (D) Percentage of nucleolar types in the total set of HCT116 cells. HCT116 p53 $3^{+/+}$(left panel) and $p 53^{-/ 2}$ (right panel) cells were examined at 24, 48 and $72 \mathrm{~h}$ after irradiation with $2 \mathrm{~Gy}$ of X-rays. The comparison of individual nucleolar types is shown in supplementary information (Fig. S2A-E). (E) Percentage of nucleolar types at 24, 48 and $72 \mathrm{~h}$ after irradiation with $0.5,1,2,4,8$, or 16 Gy of X-rays in HCT116 p53 $3^{-/}$cells. Similar results were obtained by two independent experiments. (F) Percentage of nucleolar types in the entire set of U2OS cells. Cells after transfection with control siRNA (left panel) or p53 siRNA (right panel) were harvested at 24, 48 and $72 \mathrm{~h}$ after irradiation with 2 Gy of X-rays. The comparison of individual nucleolar types is shown in the supplementary information (Fig. S2F-J). The error bars represent the standard deviation of three independent experiments. ${ }^{*} \mathrm{P}<0.05,{ }^{* *} \mathrm{P}<0.01,{ }^{* * *} \mathrm{P}<0.001$. NS, not significant, for panels A and C. IR, ionizing radiation.

By contrast, when examining nucleolar fragmentation levels in HCT116 $p 53^{+/+}$cells exogenously overexpressing p53 (Fig. 5A), it was observed that, compared with mock cells, the HCT116 $p 53^{+/+}$cells overexpressing $p 53$ exhibited a marginal increase in nucleolar fragmentation at $72 \mathrm{~h}$ after irradiation with 2 Gy of X-rays (Fig. 5B and C).

Carbon ions induce moderately greater nucleolar fragmentation compared with $X$-rays. High LET carbon ion irradiation is one of the most promising next-generation radiotherapies (20). Following high LET carbon ion irradiation, critical genome instability causes greater cell-killing effect due to the formation of complex DNA lesions and clustered DSBs, leading to large deletions or chromosomal translocations $(21,22)$. To evaluate the biological effects of high LET carbon ion irradiation on nucleolar stability, the number of nucleoli per cell and the morphology were examined after 2 Gy of X-ray or carbon ion irradiation. Similar to the results following X-ray irradiation (Fig. 1), the number of nucleoli per cell increased after carbon ion irradiation in HCT116 $p 53^{+/+}$cells and U2OS cells (Fig. 6A and $\mathrm{B})$. The increase in the number of nucleoli after carbon ion irradiation was modestly but statistically significantly higher at $24 \mathrm{~h}(\mathrm{P}=0.001)$ and at $48 \mathrm{~h}(\mathrm{P}<0.001)$ compared with that after X-ray irradiation in HCT116 $p 53^{+/+}$cells. Compared with HCT116 $p 53^{+/+}$cells, U2OS cells exhibited a modest increase in the number of nucleoli following carbon ion irradiation compared with that after X-ray irradiation; however, it was not statistically significant. Although the type distribution was similar between $\mathrm{X}$-rays and carbon ion irradiation, the morphological changes in HCT116 $p 53^{+/+}$and $\mathrm{U} 2 \mathrm{OS}$ cells were also observed after carbon ion irradiation (Fig. 6C-F, supplementary information Figs. S1C and S3A-H). Next, we examined the number of nucleoli per cell and the morphological changes under a p53 defective background following carbon ion irradiation. At $24-48 \mathrm{~h}$ after IR, there was no significant alteration in the number of nucleoli due to p53 deficiency in either HCT116 or U2OS cells, as observed after X-ray exposure (Fig. 7A and B). However, a higher percentage of type 4 morphology was found in HCT116 $p 53^{--}$cells at $72 \mathrm{~h}$ after carbon ion irradiation, compared with after X-rays or HCT116 $p 53^{+/+}$cells after carbon ion irradiation (Fig. 7C and D, supplementary information Figs. S1D and S4D). Type 6, which was rarely observed in other conditions, was detected in HCT116 $p 53^{-/}$cells after carbon ion irradiation (Fig. 7D, supplementary information Fig. S4F). By contrast, a similar type of morphological change was observed in U2OS cells irrespective of the type of IR (Fig. 7E and F, supplementary information Fig. S4G-K). In the normal human fibroblast, similar to X-ray irradiation, carbon ion irradiation did not increase the number of nucleoli (supplementary information Fig. S5). 

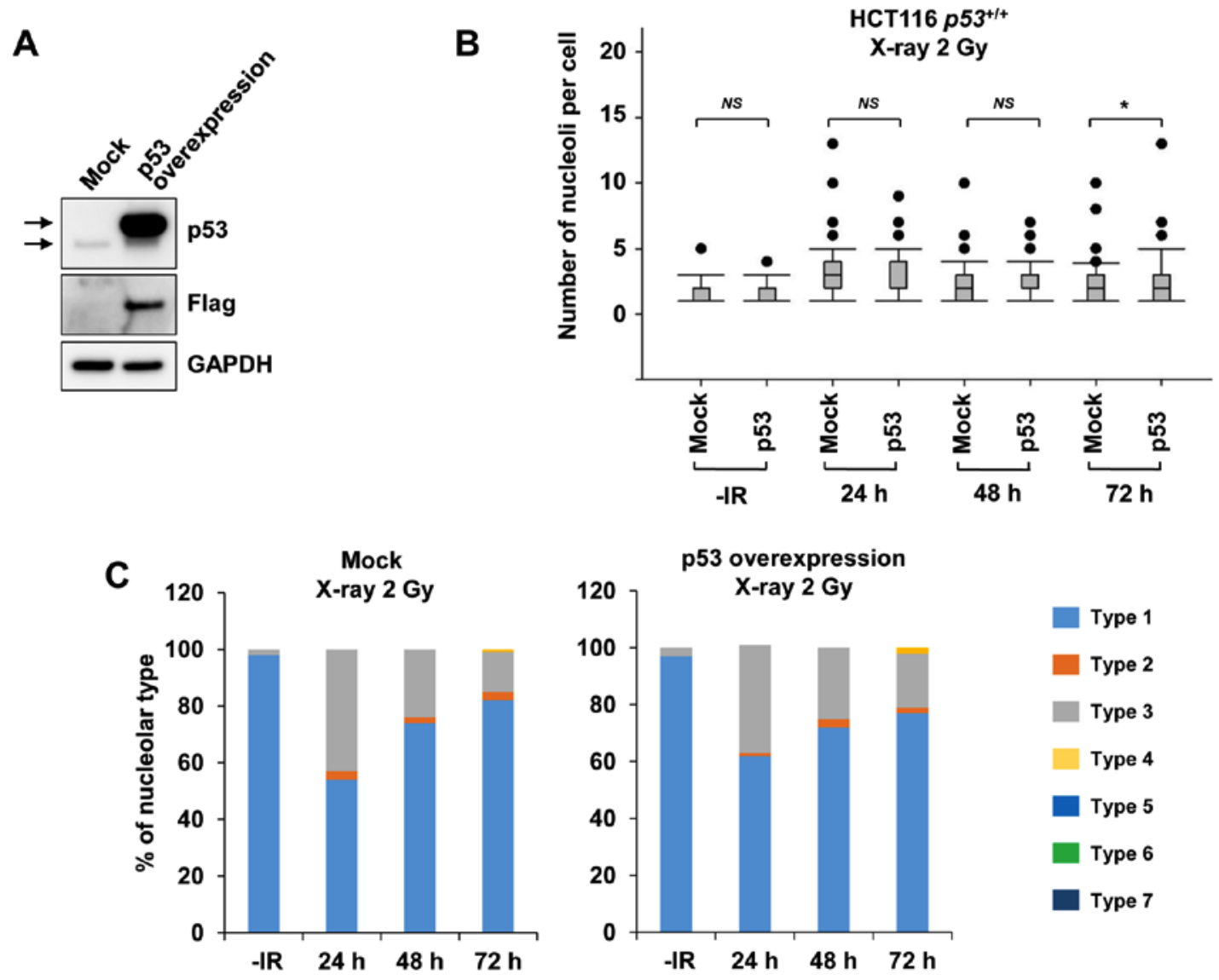

Figure 5. Overexpression of p53 marginally increases nucleolar fragmentation following X-rays. (A) p53 expression level. Flag-p53 expression vector was transfected in HCT116 $p 53^{+/+}$cells. Cells were irradiated $24 \mathrm{~h}$ after 553 transfection. (B) Distribution of the number of nucleoli per cell at 24,48 and $72 \mathrm{~h}$ after irradiation with $2 \mathrm{~Gy}$ of X-rays in HCT116 $p 53^{+/+}$mock and p53-overexpressing cells. (C) Percentage of nucleolar types in the total set of evaluated HCT116 $p 53^{+/ /}$mock and p53-overexpressing cells. Cells were harvested at 24, 48 and $72 \mathrm{~h}$ after irradiation with $2 \mathrm{~Gy}$ of X-rays. Similar results were obtained by two independent experiments. Statistical significance was examined by comparison of mock and p53-overexpressing cells at respective time points. "P $<0.05$. NS not significant; IR, ionizing radiation.

Transcriptional activity of rDNA is reduced under conditions of $p 53$ deficiency following $I R$. To address the question whether nucleolar fragmentation after IR affects transcriptional activity of rDNA, qPCR was performed to measure the level of 47S rRNA, an immediate transcript of rDNA, which ultimately yields mature rRNA after RNA processing. Importantly, consistent with the results of nucleolar fragmentation, a significant decrease in rRNA synthesis was observed in HCT116 p53 $3^{-/}$cells $72 \mathrm{~h}$ after irradiation with 4-16 Gy of X-rays; however, X-ray irradiation did not change rRNA synthesis in HCT116 $p 53^{+/+}$cells after 2, 4 or 8 Gy (Fig. 8A and B). Instead, an increase was observed in HCT116 $p 53^{+/+}$ cells after $16 \mathrm{~Gy}$.

\section{Discussion}

In the present study, it was observed that IR causes nucleolar fragmentation, suggesting that DNA damage disrupts the nucleolar structure. Furthermore, it was observed that cancer cells lacking p53 or subjected to p53 siRNA display increased nucleolar fragmentation following IR. Compared with X-ray irradiation, carbon ion irradiation, generating complex DNA lesions and clustered DSBs, caused only slightly greater nucleolar fragmentation. In addition, the analysis of $47 \mathrm{~S}$ rRNA using qPCR revealed a significant reduction of ribosomal RNA expression in p53-defective cells following IR. These results suggest that IR causes nucleolar instability, and p53 plays a key role in its prevention.

A chromosomal rearrangement, such as a large deletion, duplication, inversion or translocation, is a characteristic manifestation of dynamic genome instability following IR. The nucleolar fragmentation detected in the present study likely occurs during chromosomal rearrangements. In human cells, 150-200 copies of rDNA encoding rRNA are located on five distinct chromosomes. Each chromosome contains a repetitive rDNA sequence. As rRNA forms secondary structures, such as hairpins, stem-loops, or cruciform structures (23), such loop structures are also expected to be formed in rDNA when DNA becomes single-stranded (e.g., during DNA replication or transcription). In the present study, however, it may be inferred that DSBs at rDNA regions are unlikely to be induced by IR, since the percentage of rDNA in the total genome is $<1 \%$. For example, 2 Gy of X-rays induce $\sim 60$ DSBs per cell in G1 phase cells and $\sim 120$ DSBs in G2 phase cells (24). Therefore, only 1 or $<1$ DSB per cell are induced at the rDNA region after 2-8 Gy of X-rays. Since such isolated DSBs are unlikely to be the cause of nucleolar fragmentation, it may be inferred that additional steps are required to induce nucleolar fragmentation following 
A

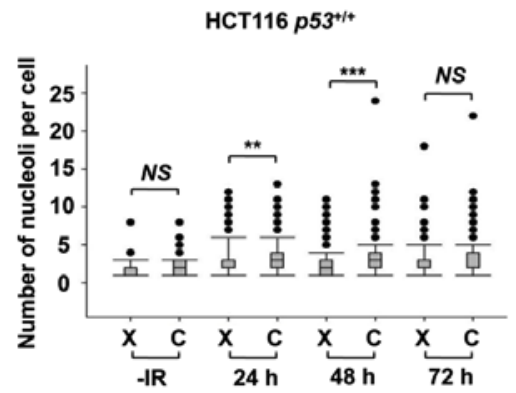

B

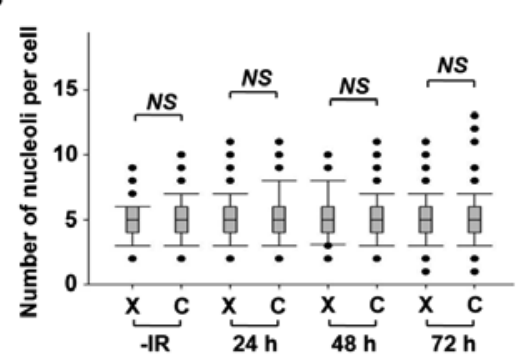

C

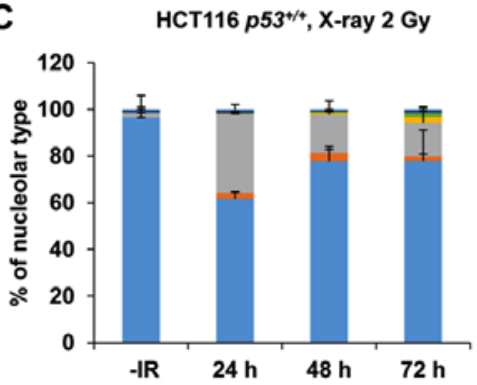

E

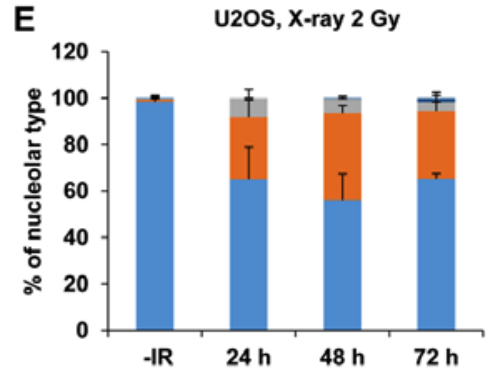

D HCT116 $p 5^{3++}$, Carbon-ion 2 Gy
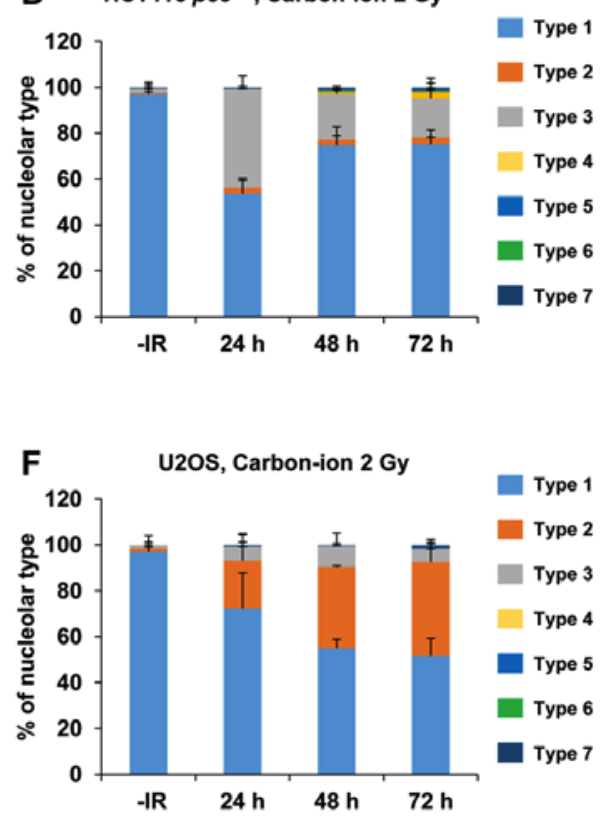

Figure 6. Carbon ions induce moderately greater nucleolar fragmentation compared with X-rays. (A and B) Distribution of the number of nucleoli per cell at 24 , 48 and $72 \mathrm{~h}$ after irradiation with $2 \mathrm{~Gy}$ of X-rays or $2 \mathrm{~Gy}$ of carbon ions in (A) HCT116 p53 ${ }^{+/+}$and (B) U2OS cells. X: X-ray, C: carbon ion. (C and D) Percentage of nucleolar types in the total set of evaluated HCT116 $p 53^{+/+}$cells. Cells were harvested at 24, 48 and 72 h after irradiation with (C) 2 Gy of X-rays or (D) 2 Gy of carbon ions. The comparison of individual nucleolar types is shown in the supplementary information (Fig. S3A-E). (E and F) Percentage of nucleolar types in the total set of evaluated U2OS cells. Cells were harvested at 24, 48 and $72 \mathrm{~h}$ after irradiation with (E) 2 Gy of X-rays or (F) 2 Gy of carbon ions. The comparison of individual nucleolar types is shown in the supplementary information (Fig. S3F-H). The statistical significance in (A and B) was examined by comparing X-ray- and carbon ion-irradiated samples at the respective time points. The error bars represent the standard deviation of three independent experiments. ${ }^{* *} \mathrm{P}<0.01,{ }^{* * *} \mathrm{P}<0.001$. NS, not significant; IR, ionizing radiation.

IR. One possibility is that DNA replication-associated DSBs in $\mathrm{S}$ phase cells may be the cause of nucleolar fragmentation. IR causes multiple types of DNA damage, such as DSBs, base damage, SSBs and cross-link damage. The amount of base damage and SSBs is estimated to be $\sim 1,000-3,000$ per cell per Gy (25). The number of direct DSBs after 1 Gy X-ray is $\sim 30$ per G1 cell (24). However, if a DNA replication fork encounters unrepaired base damage or SSBs, the damage is converted into DSBs, namely replication-associated DSBs (i.e., several thousand DSBs may be formed at replication sites) (26). The generation of DNA replication-associated DSBs may be augmented under p53 deficiency. Since p53 plays an important role in the G1/S checkpoint arrest following IR (27), the lack of a p53-dependent G1 arrest leads to the enhancement of the replication-associated DSBs. This model is supported by the findings of the present study, showing that nucleolar fragmentation following IR is augmented in p53-defective cells.

At the beginning of this study, it was expected that carbon ion irradiation may cause substantially greater nucleolar fragmentation, as it is associated with a higher frequency of chromosomal rearrangements compared with that observed after X-rays; however, the present analysis uncovered only a subtle enhancement of nucleolar fragmentation following carbon ion exposure. Replication-associated DSBs from base damage and SSBs may be the primary source of nucleolar fragmentation, as carbon ion-specific DSBs may not be implicated in the enhancement of nucleolar fragmentation. Consistent with this model, it may be inferred that p53 deficiency, which causes failure of G1/S checkpoint arrest (i.e., increase in $\mathrm{S}$ phase entry), led to nucleolar fragmentation. Additionally, G2/M checkpoint and mis-segregation during mitosis may be a major factor inducing nucleolar fragmentation. On morphological analysis, an increase in the formation of micronuclei with nucleolin signals was observed following IR, particularly in HCT116 cells and p53-depleted U2OS cells. As micronuclei are formed following mis-segregation in mitosis $(28,29)$ the sensitivity of the G2/M checkpoint arrest is associated with nucleolar fragmentation. Although the role of p53 in G2/M checkpoint arrest following IR may be less significant, the lack of p53 may promote the formation of micronuclei with nucleolin signals through mis-segregation in mitosis following inefficient G2/M checkpoint arrest, particularly in U2OS cells. Additionally, it was observed that the primary type of fragmented nucleoli following IR in HCT116 cells was type 3, whereas in U2OS cells it was type 2. Although the precise mechanism underlying such a distinct type of formation is unclear, different responses between HCT116 and U2OS cells against the G1/S and G2/M checkpoint arrest may be involved. In contrast to the results observed in cancer cell lines, the normal human fibroblasts (1BR hTERT) did not exhibit an increase in the number of nucleolar fragments. Since normal fibroblasts display an intact G1/S and G2/M checkpoint arrest, nucleolar fragmentation and formation of micronuclei may not be efficiently induced via DNA replication and mitosis, respectively. In addition, following persistent checkpoint arrest, fibroblast cells tend to undergo cellular senescence rather than apoptosis (30); these distinct characteristics may explain the different levels 
A

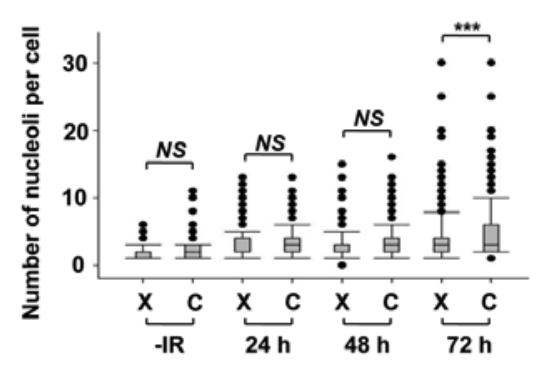

B

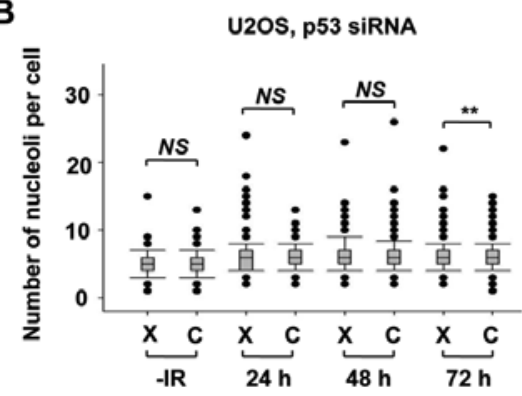

C

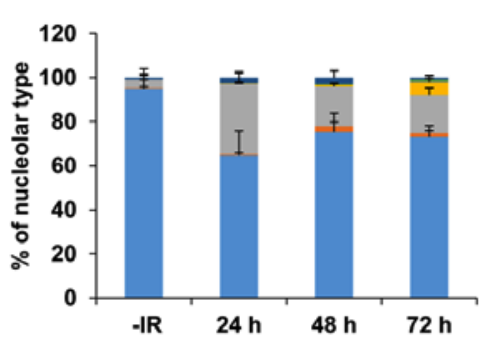

E

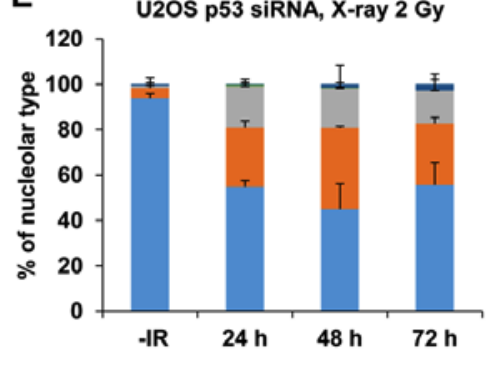

D HCT116 $p 53^{-*}$, Carbon-ion 2 Gy

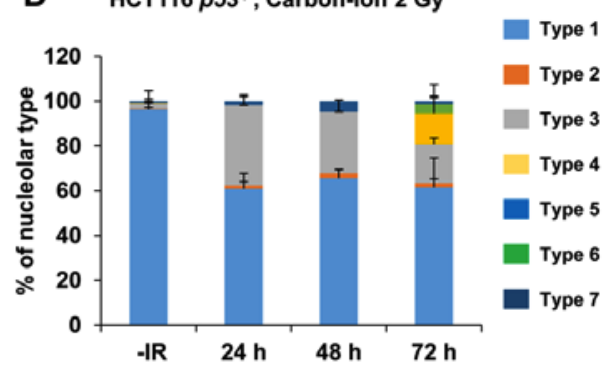

$\mathbf{F}$

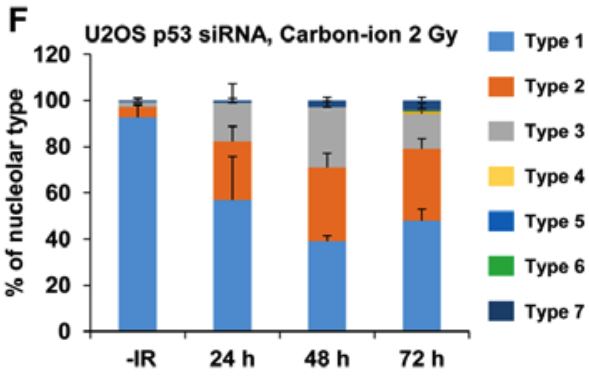

Figure 7. Carbon ion irradiation augments nucleolar fragmentation in p53-deficient cells. (A and B) The number of nucleoli per cell in (A) HCT116 $p 53^{-/-}$cells was examined at 24,48 and $72 \mathrm{~h}$ after $2 \mathrm{~Gy}$ of X-rays or 2 Gy of carbon ions. (B) Similarly, the number of nucleoli per cell in U2OS p53 siRNA cells was examined. X, X-ray; C, carbon ion. (C and D) Percentage of nucleolar types in the total set of evaluated HCT116 $p 53^{--}$cells at 24,48 and $72 \mathrm{~h}$ after irradiation with (C) 2 Gy of X-rays or (D) 2 Gy of carbon ions. The comparison of individual nucleolar types is shown in the supplementary information (Fig. S4A-F). (E and F) Percentage of nucleolar types in the total set of evaluated U2OS p53 siRNA cells. Cells were harvested at 24,48 and $72 \mathrm{~h}$ after irradiation with (E) 2 Gy of X-rays or (F) 2 Gy of carbon ions. The comparison of the individual nucleolar types is shown in the supplementary information (Fig. S4G-K). The statistical significance in A and B was examined by a comparison of X-ray- and carbon ion-irradiated samples at the respective time points. The error bars represent the standard deviation of three independent experiments. ${ }^{* *} \mathrm{P}<0.01,{ }^{* * *} \mathrm{P}<0.001$. NS, not significant; IR, ionizing radiation.

A

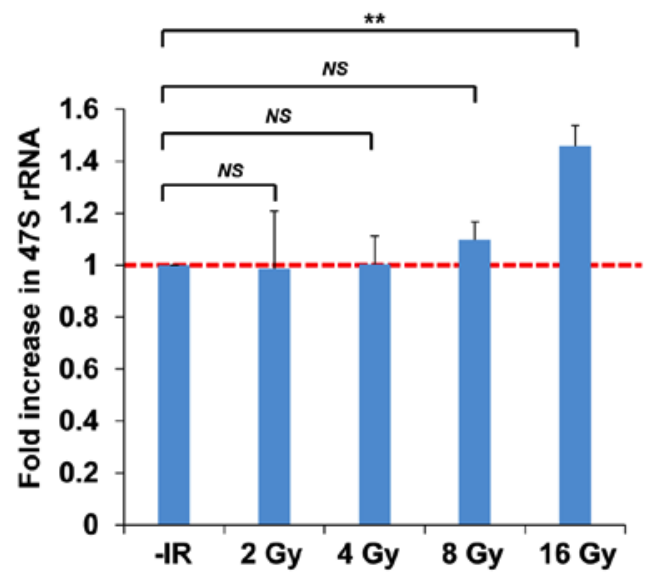

B

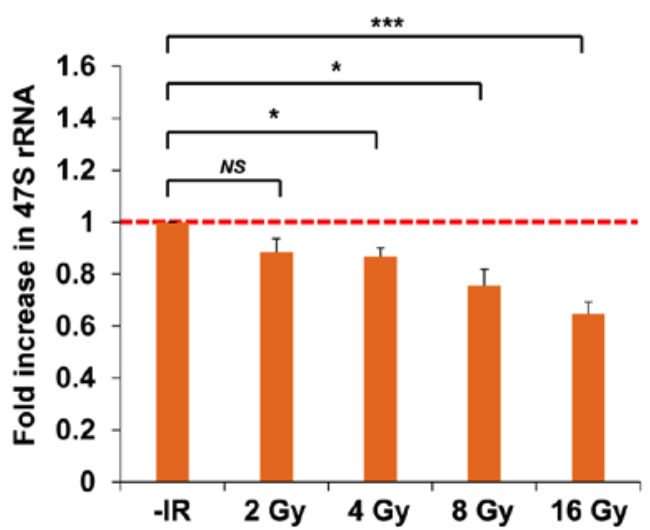

Figure 8. Transcriptional activity of rDNA is reduced under conditions of p53 deficiency following IR. (A and B) Levels of 47S precursor rRNA, quantified by qPCR analysis $72 \mathrm{~h}$ after irradiation of $\mathrm{HCT} 116$ (A) $p 53^{+++}$(B) or $p 53^{-/-}$cells with $2,4,8$, or $16 \mathrm{~Gy}$ of X-rays. The error bars represent the standard deviation of three independent experiments. The statistical significance was examined between irradiated cells at the indicated dose and non-irradiated cells, using Bonferroni's correction. ${ }^{*} \mathrm{P}<0.0125,{ }^{* *} \mathrm{P}<0.0025,{ }^{* * *} \mathrm{P}<0.00025$. NS, not significant; IR, ionizing radiation; qPCR, quantitative polymerase chain reaction.

of nucleolar fragmentation following IR. By contrast, cancer cells, which normally exhibit the predominant modes of cell death after irradiation, preferentially undergo apoptosis and mitotic catastrophe under p53 intact and deficient conditions, respectively (12). Thus, it appears that the checkpoint machinery involving G1/S and G2/M checkpoint arrest and apoptosis under the regulation of p53 plays a critical role in preventing nucleolar fragmentation following IR (Fig. 9).

In addition to nucleolar fragmentation, rRNA production was found to be reduced in p53-defective cells following IR. Since rRNA is an essential component of the ribosome, which is required for all protein synthesis, the reduction of 
Cancer cell $\left(p 53^{+/+}\right)$

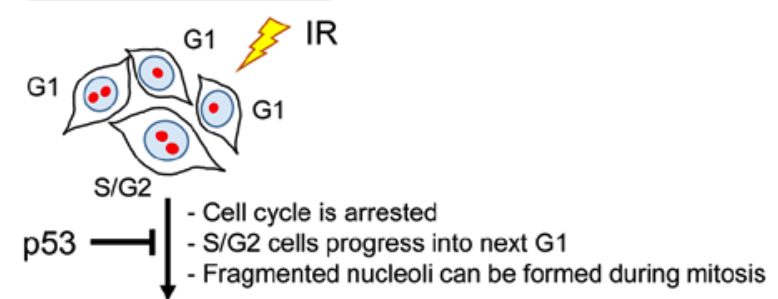

G1

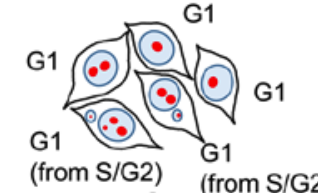

p53 $\rightarrow \mid \begin{aligned} & \text { - Irradiated S/G2 cells progress into next G1 } \\ & \text { undergo p53 dependent-apoptosis }\end{aligned}$

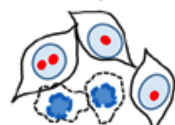

Intact rDNA transcription

in total population

\section{Cancer cell $\left(p 53^{-\sim}\right)$}
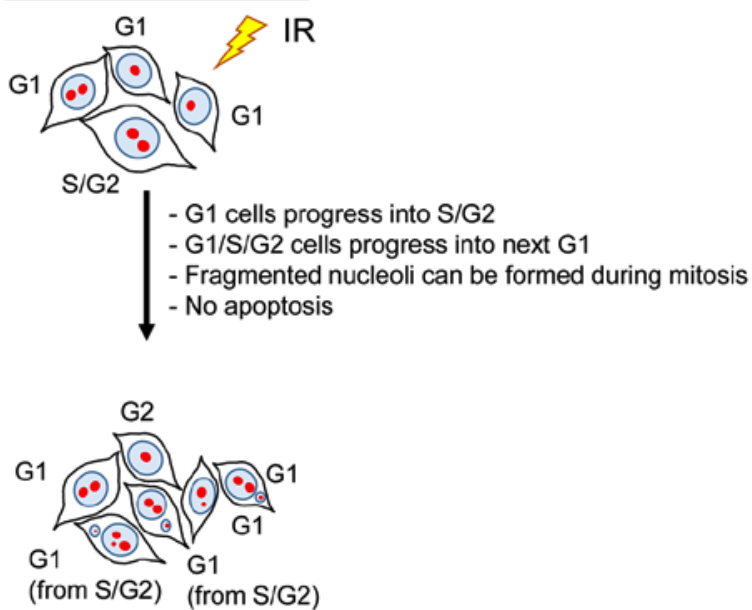

Impaired rDNA transcription

in total population

Figure 9. Model for the pathway of nucleolar instability following IR. Following activation of cell cycle checkpoint arrest after IR, irradiated cells are arrested at each cell cycle phase. Since the G1/S checkpoint arrest is sensitive, G1 cells are arrested until most DSBs are repaired, if p53 is present. G2/M checkpoint arrest is released when the number of DSBs in G2 cells becomes 10-20, and cells progress into the M phase. During mitosis, micronuclei with or without a nucleolin signal may form. After mitosis, G1 cells that have progressed from irradiated S/G2 cells may undergo p53-dependent apoptosis. Thus, surviving cells may exhibit normal transcriptional activity of rDNA. By contrast, as irradiated cells may not be effectively arrested under conditions of p53 deficiency, the formation of micronuclei is a frequent occurrence following mitosis. In addition, as p53-deficient G1 cells with micronuclei are not able to undergo apoptosis, surviving cells displaying a damaged nucleolus may exhibit low transcriptional activity of rDNA. IR, ionizing radiation, DSBs, double-strand breaks.

ribosomal biogenesis would impair cellular activity and viability. In contrast to p53-defective cells, rRNA production in p53-proficient cells was not reduced, but rather enhanced, following high-dose IR. As described above, a proportion of cells undergo mitosis due to a checkpoint release following a prolonged $\mathrm{G} 2 / \mathrm{M}$ checkpoint arrest, which results in micronuclei formation. Following mitosis, cells with micronuclei may undergo apoptosis in the next G1 phase if the p53-dependent apoptosis pathway is intact. Thus, in p53-proficient cells, normal levels of rDNA transcription may be sustained in the remaining cells with intact nucleoli, as cells with fragmented nucleoli are eliminated by apoptosis. On the other hand, in p53-deficient cancer cells, the remaining cells exhibit inefficient rDNA transcriptional activity due to the nucleolar fragmentation, as cells displaying fragmented nucleoli with micronuclei cannot efficiently undergo apoptosis.

Consistently with the present findings, evidence supporting the involvement of p53 in nucleolar biogenesis has also been provided by previous studies $(31,32)$. The downregulation of ribosome biogenesis leads to the release of ribosomal proteins, including Arf, from the nucleolus to the nucleoplasm, which promotes the binding between Murine Double Minute 2 (MDM2) and released ribosomal proteins (31). Thus, the prevention of MDM2-dependent p53 degradation results in p53 activation. In addition, nucleolin and ribosomal protein L26 (RPL26) bind to the 5' untranslated region of p53 mRNA after DNA damage, thereby controlling the translation of p53 (32). In conclusion, the present study demonstrated that p53 plays an important role in maintaining the transcriptional activity of rDNA in response to DNA damage. The downregulation of the
rDNA-rRNA axis (i.e., the pathway of ribosomal biogenesis) under conditions of p53 deficiency may be involved in cancer development via the impairment of cellular homeostasis, particularly after IR or genotoxic stress. These pathways play a significant role in the maintenance of ribosomal biogenesis as one of the mechanisms of cancer prevention, as the downregulation of the rDNA-rRNA axis may be caused by the two key functions of p53, including G1/S and G2/M checkpoint arrest and apoptosis.

\section{Acknowledgements}

The authors would like to thank Yoshimi Omi, Akiko Shibata, Yoko Hayashi, Shiho Nakanishi, Yukihiko Yoshimatsu, Itaru Sato and Yuka Hirota for assisting with the laboratory work.

\section{Funding}

The present study was supported by Grants-in-Aid from the Japan Society for the Promotion of Science for KAKENHI (JP17H04713 to AS), the Takeda Science Foundation, the Uehara Memorial Foundation, and the Program of the network-type Joint Usage/Research Center for Radiation Disaster Medical Science of Hiroshima University, Nagasaki University and Fukushima Medical University. The present study was also supported by Grants-in-Aid from the Ministry of Education, Culture, Sports, Science and Technology of Japan for programs for Leading Graduate Schools, Cultivating Global Leaders in Heavy Ion Therapeutics and Engineering. 


\section{Availability of materials and data}

All the datasets generated and analyzed during the present study are available from the corresponding author on reasonable request.

\section{Authors' contributions}

AS designed the experiments and wrote the paper with SK. The experiments including immunofluorescence, immunoblotting and qPCR were performed by SK, YH and AS. Acquired data were analyzed and interpreted by SK, WG, YH, TO, HS, MY, RK, TY and AS. The manuscript was reviewed by TO, HS, KDH, MY and TY. Administrative, technical, or material support was provided by TO, SL and TN. The study was supervised by AS.

\section{Ethics approval and consent to participate}

Not applicable.

\section{Patient consent for publication}

Not applicable.

\section{Competing interests}

The authors declare that they have no conflict of interest.

\section{References}

1. Buchwalter A and Hetzer MW: Nucleolar expansion and elevated protein translation in premature aging. Nat Commun 8: 328, 2017.

2. Ruggero D: Revisiting the nucleolus: From marker to dynamic integrator of cancer signaling. Sci Signal 5: pe38, 2012.

3. Montanaro L, Trere D and Derenzini M: Changes in ribosome biogenesis may induce cancer by down-regulating the cell tumor suppressor potential. Biochim Biophysica Acta 1825: 101-110, 2012.

4. Stults DM, Killen MW, Williamson EP, Hourigan JS, Vargas HD, Arnold SM, Moscow JA and Pierce AJ: Human rRNA gene clusters are recombinational hotspots in cancer. Cancer Res 69: 9096-9104, 2009.

5. Negi SS and Brown P: rRNA synthesis inhibitor, CX-5461, activates ATM/ATR pathway in acute lymphoblastic leukemia, arrests cells in G2 phase and induces apoptosis. Oncotarget 6: 18094-18104, 2015.

6. Shibata A and Jeggo P: A historical reflection on our understanding of radiation-induced DNA double strand break repair in somatic mammalian cells; interfacing the past with the present. Int J Radiat Biol 95: 945-956, 2019.

7. van Gent DC, Hoeijmakers JH and Kanaar R: Chromosomal stability and the DNA double-stranded break connection. Nat Rev Genet 2: 196-206, 2001.

8. Hada $\mathrm{M}$ and Georgakilas AG: Formation of clustered DNA damage after high-LET irradiation: A review. J Radiat Res 49: 203-210, 2008

9. Nakajima NI, Brunton H, Watanabe R, Shrikhande A, Hirayama R, Matsufuji N, Fujimori A, Murakami T, Okayasu R, Jeggo P and Shibata A: Visualisation of gammaH2AX foci caused by heavy ion particle traversal; distinction between core track versus non-track damage. PLoS One 8: e70107, 2013.

10. Hagiwara Y, Niimi A, Isono M, Yamauchi M, Yasuhara T, Limsirichaikul S, Oike T, Sato H, Held KD, Nakano T and Shibata A: 3D-structured illumination microscopy reveals clustered DNA double-strand break formation in widespread $\gamma \mathrm{H} 2 \mathrm{AX}$ foci after high LET heavy-ion particle radiation. Oncotarget 8: 109370-109381, 2017.
11. Hagiwara Y, Oike T, Niimi A, Yamauchi M, Sato $H$, Limsirichaikul S, Held KD, Nakano T and Shibata A: Clustered DNA double-strand break formation and the repair pathway following heavy-ion irradiation. J Radiat Res 60: 69-79, 2019.

12. Amornwichet N, Oike T, Shibata A, Ogiwara H, Tsuchiya N, Yamauchi M, Saitoh Y, Sekine R, Isono M, Yoshida Y, et al: Carbon-ion beam irradiation kills X-ray-resistant p53-null cancer cells by inducing mitotic catastrophe. PLoS One 9: e115121, 2014.

13. Durante M and Cucinotta FA: Heavy ion carcinogenesis and human space exploration. Nat Rev Cancer 8: 465-472, 2008.

14. Henderson AS, Warburton D and Atwood KC: Location of ribosomal DNA in the human chromosome complement. Proc Natl Acad Sci USA 69: 3394-3398, 1972.

15. Durkin SG and Glover TW: Chromosome fragile sites. Annu Rev Genet 41: 169-192, 2007.

16. Montgomery PO, Karney D, Reynolds RC and McClendon D: Cellular and subcellular effects of ionizing radiations. Am J Pathol 44: 727-746, 1964.

17. Hidvegi EJ, Holland J, Boloni E, Lonai P, Antoni F and Varteresz V: The effect of whole-body x-irradiation of guinea pigs on liver ribosomes. Biochem J 109: 495-505, 1968.

18. Shibata A, Conrad S, Birraux J, Geuting V, Barton O, Ismail A, Kakarougkas A, Meek K, Taucher-Scholz G, Löbrich M and Jeggo PA: Factors determining DNA double-strand break repair pathway choice in G2 phase. EMBO J 30: 1079-1092, 2011.

19. Livak KJ and Schmittgen TD: Analysis of relative gene expression data using real-time quantitative PCR and the 2(-Delta Delta C(T)) method. Methods 25: 402-408, 2001.

20. Ohno T: Particle radiotherapy with carbon ion beams. EPMA J 4: 9, 2013.

21. Oike T, Niimi A, Okonogi N, Murata K, Matsumura A, Noda SE, Kobayashi D, Iwanaga M, Tsuchida K, Kanai T, et al: Visualization of complex DNA double-strand breaks in a tumor treated with carbon ion radiotherapy. Sci Rep 6: 22275, 2016.

22. Niimi A, Yamauchi M,Limsirichaikul S, Sekine R, Oike T, Sato H, Suzuki K, Held KD, Nakano T and Shibata A: Identification of DNA double strand breaks at chromosome boundaries along the track of particle irradiation. Genes Chromosomes Cancer 55: 650-660, 2016

23. Petrov AS, Bernier CR, Gulen B, Waterbury CC, Hershkovits E, Hsiao C, Harvey SC, Hud NV, Fox GE, Wartell RM and Williams LD: Secondary structures of rRNAs from all three domains of life. PLoS One 9: e88222, 2014.

24. Lobrich M, Shibata A, Beucher A, Fisher A, Ensminger M, Goodarzi AA, Barton O and Jeggo PA: gammaH2AX foci analysis for monitoring DNA double-strand break repair: Strengths, limitations and optimization. Cell Cycle 9: 662-669, 2010.

25. Powell S and McMillan TJ: DNA damage and repair following treatment with ionizing radiation. Radiother Oncol 19: 95-108, 1990.

26. Vilenchik MMand Knudson AG: Endogenous DNA double-strand breaks: Production, fidelity of repair, and induction of cancer. Proc Natl Acad Sci USA 100: 12871-12876, 2003.

27. Amundson SA, Myers TG and Fornace AJ Jr: Roles for p53 in growth arrest and apoptosis: Putting on the brakes after genotoxic stress. Oncogene 17: 3287-3299, 1998.

28. Fenech M, Kirsch-Volders M, Natarajan AT, Surralles J, Crott JW, Parry J, Norppa H, Eastmond DA, Tucker JD and Thomas P: Molecular mechanisms of micronucleus, nucleoplasmic bridge and nuclear bud formation in mammalian and human cells. Mutagenesis 26: 125-132, 2011.

29. Huang H, Fletcher L, Beeharry N, Daniel R, Kao G, Yen TJ and Muschel RJ: Abnormal cytokinesis after x-irradiation in tumor cells that override the G2 DNA damage checkpoint. Cancer Res 68: 3724-3732, 2008.

30. Johmura Y, Shimada M, Misaki T, Naiki-Ito A, Miyoshi H, Motoyama N, Ohtani N, Hara E, Nakamura M, Morita A, et al: Necessary and sufficient role for a mitosis skip in senescence induction. Mol Cell 55: 73-84, 2014.

31. James A, Wang Y, Raje H, Rosby R and DiMario P: Nucleolar stress with and without p53. Nucleus 5: 402-426, 2014.

32. Takagi M, Absalon MJ, McLure KG and Kastan MB: Regulation of p53 translation and induction after DNA damage by ribosomal protein L26 and nucleolin. Cell 123: 49-63, 2005.

This work is licensed under a Creative Commons Attribution-NonCommercial-NoDerivatives 4.0 International (CC BY-NC-ND 4.0) License. 